\title{
Studies of baryonic matter at the BM@N and MPD experiments at Nuclotron/NICA
}

\section{Mikhail Kapishin* Joint Institute for Nuclear Research, 141980 Russia, Moscow region, Dubna, Joliot Curie 6}

E-mail: kapishinejinr.ru

The NICA (Nuclotron-based Ion Collider fAcility) project is under realization at the Joint Institute for Nuclear Research (JINR, Dubna). The main goal of the project is an experimental study of hot and dense strongly interacting matter in heavy ion (up to $\mathrm{Au}$ ) collisions at center-of-mass energies up to $11 \mathrm{GeV}$ per nucleon. The physics program will be performed at two experiments, BM@N (Baryonic Matter at Nuclotron) at beams extracted from the Nuclotron, and MPD (Multi-Purpose Detector) at the NICA collider. The aim of the BM@N experiment is to study interactions of relativistic heavy ion beams with fixed targets. The scientific program comprises studies of nuclear matter in the intermediate energy range between experiments at the SIS and NICA/FAIR facilities. The BM@N experiment has recorded first experimental data in the carbon, argon and krypton beams of kinetic energy per nucleon ranging from 2.3 to $4.5 \mathrm{GeV}$ per nucleon. The first measurement of short range correlations of nucleons in carbon nucleus was performed in inverse kinematics with the carbon beam and liquid hydrogen target. The MPD detector is under construction to study hot and baryon rich QCD matter in heavy ion collisions at the NICA collider in the energy range $\sqrt{s_{N N}}=4 \div 11 \mathrm{GeV}$. Physics program includes the study of collective phenomena, $\Lambda$ polarization, dilepton, hyperon and hypernuclei production under extreme conditions of highest baryonic density.

Corfu Summer Institute 2018 "School and Workshops on Elementary Particle Physics and Gravity" (CORFU2018)

31 August - 28 September, 2018

Corfu, Greece

${ }^{*}$ Speaker. 


\section{Nuclotron and NICA heavy-ion physics program}

Collisions of relativistic heavy ions provide a unique opportunity to study nuclear matter at extreme densities and temperatures. In such collisions, nuclear matter is heated and compressed for a very short amount of time (few $\mathrm{fm} / \mathrm{c})$. At the Nuclotron with beam kinetic energy per nucleon ranging from 1 to $4.5 \mathrm{GeV}$, baryons form the majority of the products in a nucleus-nucleus collision, in contrast to collisions that occur at higher energies at the RHIC or SPS accelerators. According to the Quark Gluon String Model (QGSM) calculations presented in [1], at the Nuclotron energies the nucleon density in a fireball created by two colliding gold nuclei is 3-4 times higher than the saturation density. At such densities, nucleons start to overlap. At the Nuclotron, the experimental research is focused on studies of hadrons with strangeness produced in the collision and not present in the initial state of two colliding nuclei, unlike the nucleons consisting of light ( $u$ and $d$ ) quarks. The measured yields of light and strange mesons, hyperons, and antihyperons are shown in Fig. 1 (left) as a function of the energy per nucleon-nucleon collision in the center of mass system (c.m.s) in $\mathrm{Au}+\mathrm{Au} / \mathrm{Pb}+\mathrm{Pb}$ collisions. The energy range of heavy ion beams at the Nuclotron corresponds to $\sqrt{s_{N N}}=2.3 \div 3.5 \mathrm{GeV}$, these energies are high enough to study strange mesons and multi-strange hyperons produced in nucleus-nucleus collisions close to the kinematic threshold. Heavy ion collisions are an abundant source of strange hadrons that can bind with nu-
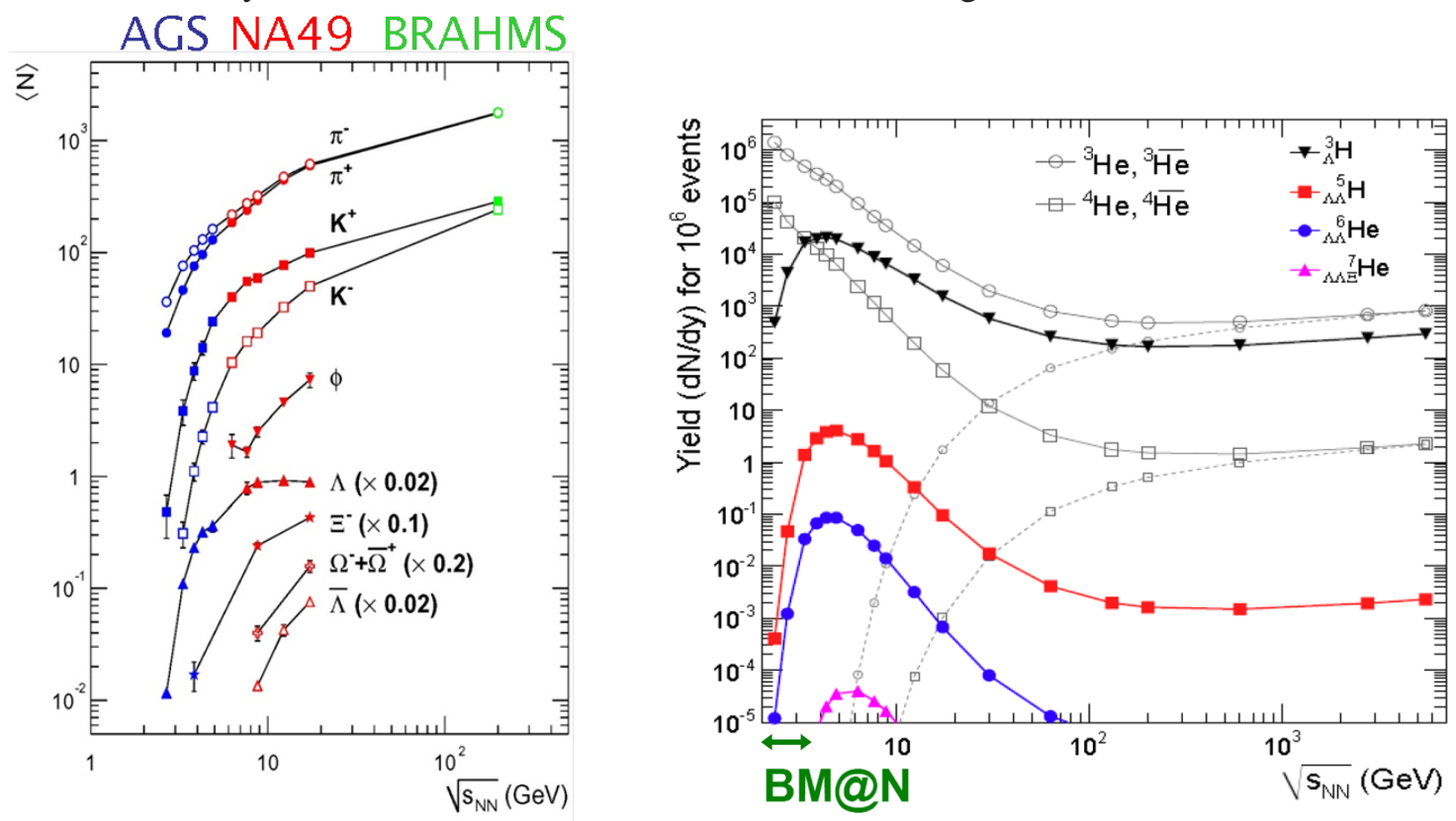

Figure 1: Left plot: Yields of mesons and (anti-)hyperons measured in different experiments as a function of the energy per nucleon-nucleon collision in c.m.s. for $\mathrm{Au}+\mathrm{Au}$ and $\mathrm{Pb}+\mathrm{Pb}$ collisions [2]. The Nuclotron beam energy range corresponds to $\sqrt{s_{N N}}=2.3 \div 3.5 \mathrm{GeV}$. Right plot: Yields of hyper-nuclei predicted by the thermal model in [3] as a function of the nucleon-nucleon collision energy in c.m.s. for $\mathrm{Au}+\mathrm{Au}$ collisions. Predictions for the yields of ${ }^{3} \mathrm{He}$ and ${ }^{4} \mathrm{He}$ nuclei are presented for comparison. The Nuclotron BM@N energy range is specified. The NICA/MPD energy range is $\sqrt{s_{N N}}=4 \div 11 \mathrm{GeV}$.

cleons and initiates formation of a variety of light hypernuclei $[4,5]$. Studies of hypernucleus production processes are expected to provide insight into the properties of hyperon-nucleon and hyperon-hyperon interactions. In Fig. 1 (right) the hypernucleus yields per event are presented as a 
function of the nucleon-nucleon collision energy in c.m.s in $\mathrm{Au}+\mathrm{Au}$ collisions as predicted by the thermal model [3]. The maximum probability of hypernucleus production is predicted for energies of $\sqrt{s_{N N}}=4 \div 5 \mathrm{GeV}$, that is close to the Nuclotron energy range and accessible at the NICA/MPD experiment. In general, the research program of the BM@N experiment aims to study heavy ion collisions at the Nuclotron including the following topics: exploration of the reaction dynamics and the equation of state $(\mathrm{EOS})$ of nuclear matter, study of in-medium properties of hadrons,, production of (multi)strange hyperons in the vicinity of the threshold and searches for hypernuclei $[6,7,8]$.

Nuclotron-based Ion Collider fAcility (NICA) is presently under the construction [18, 19]. The Multi-Purpose Detector (MPD) [20, 21] is a set-up which will be operated at the NICA collider. The NICA physics program with heavy ions will address the following topics [22]: the Equation-Of-State (EOS) of nuclear matter under extreme conditions, properties of the deconfinement phase transition, collective effects in strongly interacting matter, critical phenomena, and search for signals of chiral symmetry restoration. Both nucleus-nucleus and nucleon-nucleon interactions will be studied over a wide pseudorapidity interval and down to almost zero transverse momenta. The dense nuclear matter EOS plays a central role in the dynamics of core collapse supernova, the stability of neutron stars, and the dynamics of neutron stars mergers. The relevant signatures to be measured in heavy-ion interactions are the collective flow of identified particles, including multi-strange hyperons and hypernuclei production. At the NICA energy range a highest net-baryon density is achieved [23]. Moreover, this maximum is accompanied by the largest strangeness-to-entropy ratio in central collisions. Under such experimental conditions the formation of hypernuclei (i.e. bound states of nucleons and at least one hyperon) is enhanced, thus, for such kind of studies, a high detector capability in secondary vertices reconstruction is crucial. In addition, MPD will allow to explore bulk properties of dense baryonic matter by measuring particle yields, ratios, and transverse momentum spectra. A more precise determination of the excitation function of higher order cumulants of conserved charges (baryon number, strangeness, and charge) are important for the QCD Critical End Point search, which will provide a crucial signature of the QCD phase transition. The complete spectrum of dileptons can be accessed at NICA/MPD allowing the study of in-medium modification of vector meson properties and of fireball early phases. A rapid increase of the (anti)Lambda polarization implies the creation in heavy-ion collisions at NICA energies, of the medium with a high density vortex pattern [24, 25].

\section{BM@N detector for studies of baryonic matter at the Nuclotron}

BM@N (Baryonic Matter at Nuclotron) is the first experiment operational at the Nuclotron/ NICA accelerating complex. The purpose of the BM@N experiment is to study relativistic heavy ion beam interactions with fixed targets [7]. The Nuclotron will provide the experiment with beams of a variety of particles, from protons to gold ions, with a kinetic energy ranging from 1 to 6 $\mathrm{GeV} /$ nucleon. The maximum kinetic energy of ions with the charge to atomic weight ratio of 0.5 is $6 \mathrm{GeV} /$ nucleon. The maximum kinetic energy of gold ions with $\mathrm{Z} / \mathrm{A}$ of 0.4 is $4.5 \mathrm{GeV} /$ nucleon, while the maximum kinetic energy for protons is $13 \mathrm{GeV}$. Recently the BM@N experiment collected data in beams of carbon, argon, and krypton ions. The planned intensity of the gold ion beam at BM@N is $10^{6}$ ions/s. The acceleration of the gold ion beam is planned in 2021, after the Nuclotron upgrade. In Fig. 2 (left) the interaction rates are presented for different experiments 
with heavy ion collisions at different energies per nucleon-nucleon collision in the center of mass system. The beam energy of the BM@N experiment is in the intermediate range between experiments at the SIS and NICA/FAIR facilities and partially overlaps the energy range of the HADES experiment. The acquisition rate of non-peripheral collisions, i.e., central or intermediate interactions is expected to range from 20 to $50 \mathrm{kHz}$ at the second stage of the BM@N experiment in 2022 and later. The interaction rate is limited by the capacity of the data acquisition system and readout electronics.
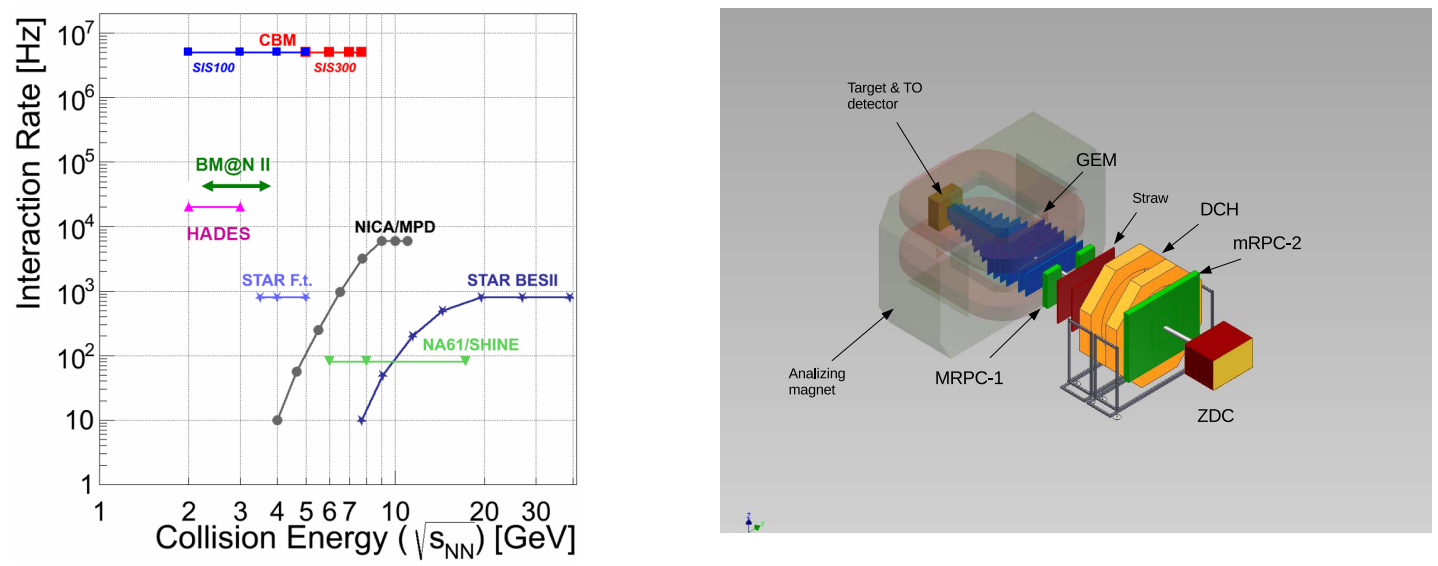

Figure 2: Left plot: Interaction rate and energy per nucleon-nucleon collision in c.m.s. in experiments with heavy ions [9]. The range for BM@N is superimposed. Right plot: Schematic view of the BM@N setup.

The layout of the proposed BM@N configuration is shown in Fig. 2 (right). The experiment combines high precision measurement of track parameters with time-of-flight information for particle identification and presumes a measurement of the total energy by the hadron calorimeter to analyze the collision centrality. The charged track momentum and multiplicity will be measured using a set of 7 planes of two-coordinate GEM (Gaseous Electron Multiplier) detectors mounted downstream of the target inside of the analyzing magnet. The GEM detectors are operational at high particle densities and in strong magnetic fields. The vertical gap between the poles of the analyzing magnet for detector installation is about $1 \mathrm{~m}$. The magnetic field can reach a maximum value of 1.2 T, which makes it possible to optimize the BM@N geometrical acceptance and resolution on momentum for different processes and energies of the beam. The outer tracking system consists of large drift chambers $(\mathrm{DCH})$ that will be supplemented with cathode strip chambers to increase the effectiveness of track measurement in Au+Au collisions. The time-of-flight detectors based on the multi-gap Resistive Plate Chamber (mRPC) technologies with strip readout provide an opportunity to separate hadrons $(\pi, \mathrm{K}, \mathrm{p})$ and light nuclei with momentum up to few $\mathrm{GeV} / \mathrm{c}$. The Zero Degree Calorimeter (ZDC) detector is foreseen for the extraction of the collision impact parameter (centrality) by measuring the energy of the fragments of colliding particles. The T0 detector positioned around the target and partially overlapping the backward hemisphere is planned to generate a trigger signal for the data acquisition and a starting signal (T0) for the time-of-flight detectors. In 2022, at the second stage of the BM@N experiment, at least four planes of two-coordinate silicon strip detectors will be installed in front of the GEM detectors to improve track reconstruction in $\mathrm{Au}+\mathrm{Au}$ collisions. Detectors of this type are currently under development for the CBM experiment [10], therefore the actualimplementation of the BM@N tracker upgrade depends on the timetable of the 
CBM silicon tracker program.

\section{First results of the $B M @ N$ technical runs}

The technical runs with the BM@N detector were performed in the deuteron beam in December 2016 and in the carbon beam in March 2017. The kinetic energy was $4 \mathrm{GeV/nucleon}$ for the deuteron beam and was varied from 3.5 to $4.5 \mathrm{GeV} /$ nucleon for the carbon beam. The starting configuration of the central tracker was based on a forward silicon strip detector and a set of GEM detectors [11]. The experimental data from the central tracker, outer drift chambers, timeof-flight detectors, zero degree calorimeter and trigger detectors were read out using the integrated data acquisition system. The collected data were used to check efficiencies of sub-detectors and develop algorithms for the event reconstruction and analysis. In particular, experimental data of minimum bias interactions of the beam with different targets were analyzed with the aim to reconstruct tracks, primary and secondary vertices using the central tracking detectors [12, 13, 14]. The track reconstruction method was based on the so-called 'cellular automaton' approach [15]. Since the GEM tracker configuration was tuned to measure relatively high-momentum beam particles, the geometrical acceptance for relatively soft decay products of strange V0 particles was rather low. The Monte Carlo simulation showed that only $\sim 4 \%$ of $\Lambda$ hyperons and $\sim 0.8 \%$ of $K_{s}^{0}$ could be reconstructed. $\Lambda$-hyperons were reconstructed using their decay mode into $p, \pi^{-}$pairs [16]. Since particle identification at this stage of the analysis was not used, all positive tracks were considered as protons and all negative as $\pi^{-}$. The invariant mass distributions of $p$ and $\pi^{-}$are shown in Fig. 3 for reconstructed interactions of the deuteron and carbon beams with the targets. The background under the signal will be reduced by introducing additional silicon tracking detectors to improve the primary and decay vertex resolution.
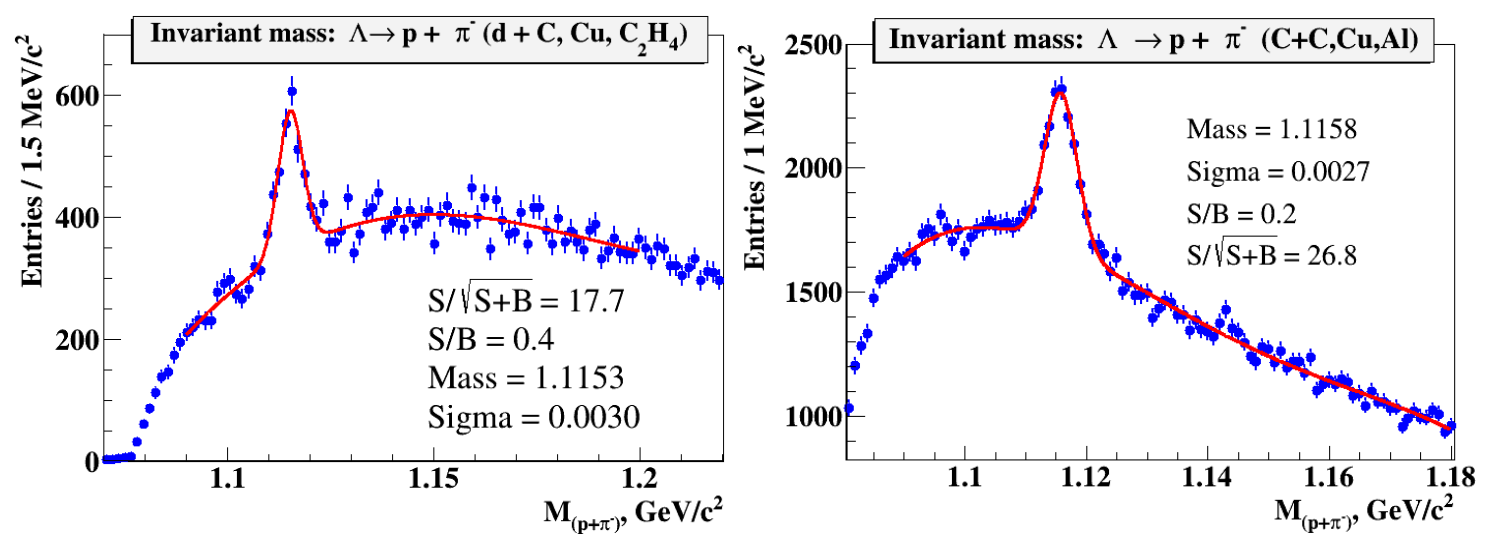

Figure 3: Invariant mass spectrum of proton and $\pi^{-}$pairs reconstructed in interactions of the deuteron beam (left plot) and carbon beam (right plot) with the targets.

The extended configuration of the BM@N set-up was realized in the recent runs with the argon and krypton beams performed in March 2018. The set-up comprised GEM detectors with the size of $163 \times 45 \mathrm{~cm}^{2}$, forward silicon strip detectors, full time-of-light system, extended trigger system, hadron and electro-magnetic calorimeters. The first measurement of short range correlations of nucleons in carbon nucleus was performed in inverse kinematics with the carbon beam and liquid hydrogen target [17]. 


\section{The MPD detector at the NICA collider}

The NICA accelerator complex includes: a heavy-ion source and heavy-ion linear accelerator, the Nuclotron - superconducting synchrotron (after the upgrade it will provide beams of heavy ions up to $6 \mathrm{~A} \mathrm{GeV}$ for isospin-symmetric nuclei and $4.5 \mathrm{~A} \mathrm{GeV}$ for $\mathrm{Au}^{79+}$ ), a booster accelerator, and two storage rings of the NICA collider. The NICA facility will provide interactions of ions up to the heaviest $\mathrm{Au}^{79+}$ with a center-of-mass energy range of $\sqrt{s_{N N}}=4 \div 11 \mathrm{GeV}$ and with a luminosity of $10^{27} \mathrm{~cm}^{-2} \mathrm{c}^{-1}$. The collider storage rings have a circumference of $503 \mathrm{~m}$, and two interaction points (IP) with MPD and SPD (Spin Physics Detector) set-ups. Until now, a substantial progress is being achieved in the construction of the NICA accelerator complex. A new heavy-ion source and heavy-ion linear accelerator have been put into operation; all the magnet elements for the NICA booster are fabricated and have passed cryogenic tests. The assembling of the booster started in the beginning of 2019. The construction of the NICA infrastructure is progressing well: the MPD building will be ready for equipment installation in the middle of 2019; the cryogenic, water cooling, and electricity systems of the NICA complex have been also substantially upgraded.

In order to fulfill the project physics goals the experimental setup at NICA should provide a large phasespace coverage as well as good tracking and particle identification capabilities. A cut-away schematic view of the MPD detector is shown in Fig.4 (left). All the MPD sub-detectors are placed inside a large superconducting solenoid, which generates a magnetic field B up to 0.5 T. In order to ensure precise track reconstruction, high magnetic field homogeneity within the solenoid's working volume will be provided by means of dedicated trim coils in the magnet poles (field inhomogeneity less than $|\Delta B| /\left|B_{0}\right| \sim 3 \cdot 10^{-4}$ ).

The main tracking system of the experiment (Time-Projection Chamber - TPC) with full azimuthal coverage will provide up to 53 space measurements per track over the pseudorapidity range $|\eta|<1$.6. The identification of charged particles within the TPC acceptance will be performed by means of ionization energy loss $(\mathrm{dE} / \mathrm{dx})$ measurements. The MPD Time-of-Flight (TOF) system will be based on Multi-gap Resistive Plate Chambers (MRPC).With the time resolution below 70 ps, the TOF system allows kaon/pion separation up to $1.7 \mathrm{GeV} / \mathrm{c}$ and up to $3 \mathrm{GeV} / \mathrm{c}$ for proton/kaon. The Electromagnetic CALorimeter (ECAL) comprises about 43000 lead-scintillator towers with the front face of $4 \times 4 \mathrm{~cm}^{2}$. The geometry of the ECAL towers provides an uniform detector performance for the measured value of electrons and gammas energy over a wide pseudorapidity range. Two arms of the Forward Hadron Calorimeter (FHCAL) positioned at either end of the MPD will be used as an event plane detector and will provide a measurement of the collision centrality. Two arrays of quartz Cherenkov counters (Fast Forward Detector - FFD) located symmetrically with respect to the MPD center will provide fast trigger signals. The MPD detector will be put into operation in two stages. The MPD configuration for the first stage which will include TPC, barrel TOF, barrel ECAL, FHCAL, and FFD sub-systems is presented in Fig. 4 (right).

\section{Feasibility studies of the MPD physics performance}

The performance of this MPD set-up for the hyperon reconstruction has been studied in minimum bias $\mathrm{Au}+\mathrm{Au}$ collisions at $\sqrt{s_{N N}}=11 \mathrm{GeV}$. A full simulation, reconstruction, and analysis chain includes particle propagation through the detector with the GEANT3 package, followed by 

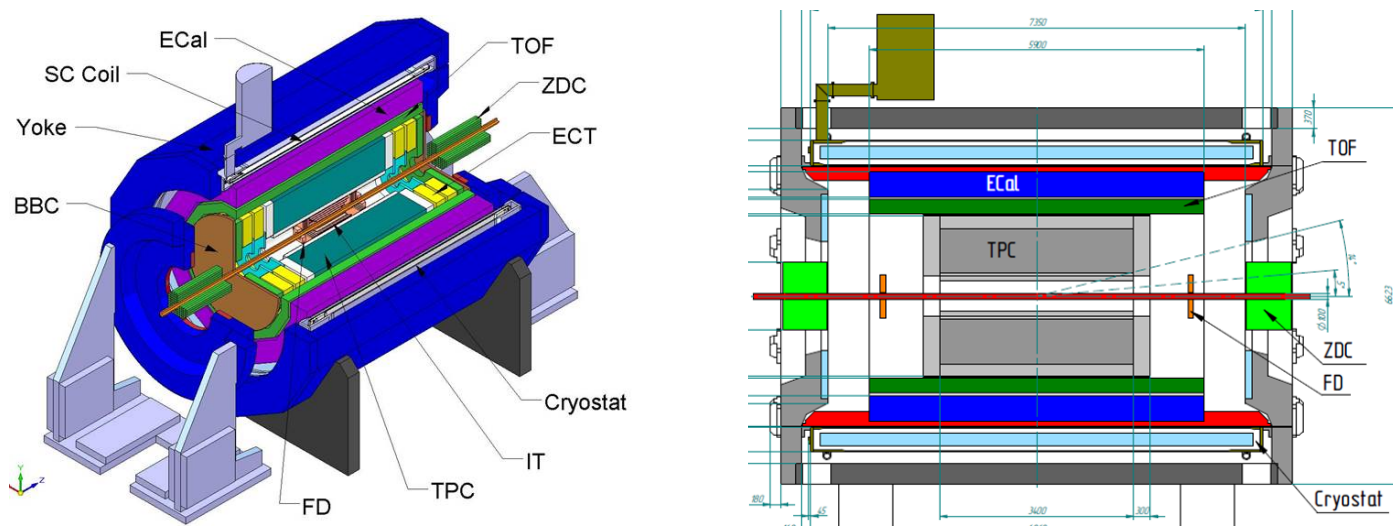

Figure 4: Schematic layout of the Multi-Purpose Detector with the complete set of detectors (left) and for the first stage of the experiment (right).

detector response simulation, track reconstruction with the Kalman filtering method, and particle identification based on the Bayesian approach for the combination of $\mathrm{dE} / \mathrm{dx}$ and TOF information. The accuracy of the primary vertex reconstruction based on the TPC charged track multiplicity varied from $100 \mu \mathrm{m}$ for central collisions to about $500 \mu \mathrm{m}$ in peripheral collisions. For the recon-
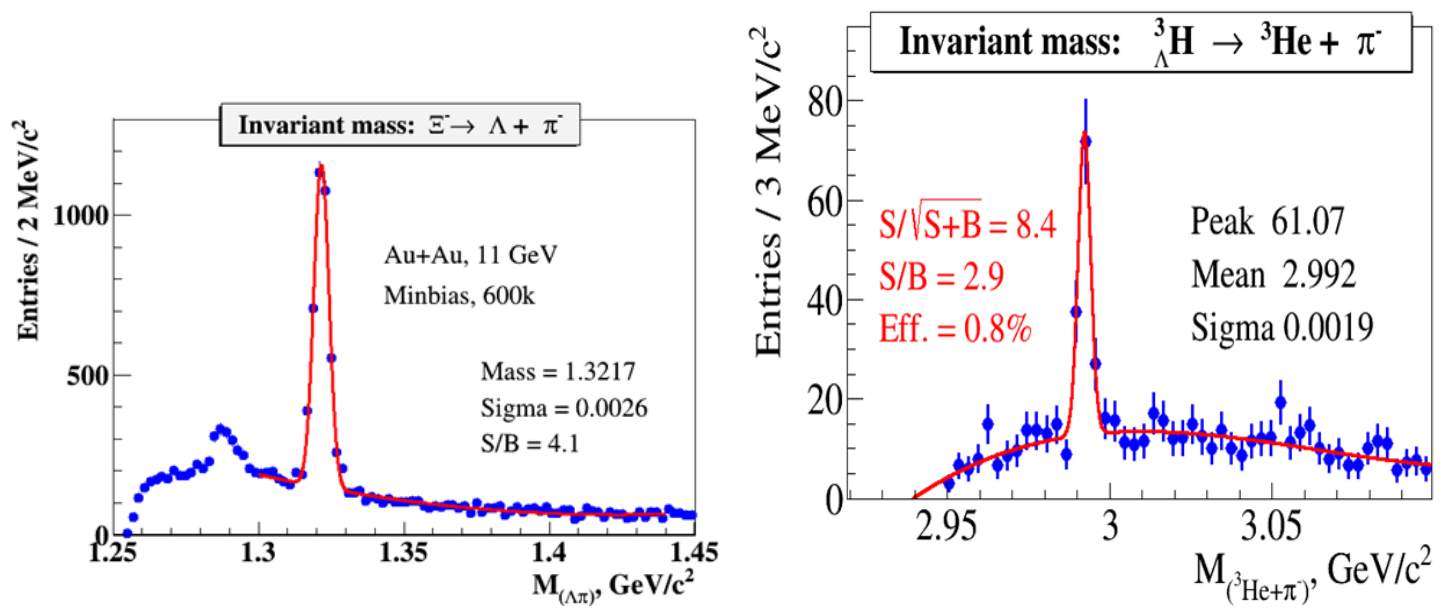

Figure 5: Simulation plots of the mass spectra of reconstructed decays of $\Xi^{-} \rightarrow \Lambda+\pi^{-}$(left); and hyper nuclei $H_{\Lambda}^{3} \rightarrow H e^{3}+\pi^{-}$(right)

struction of $\Lambda$-hyperons all tracks identified as protons are combined with negatively charged pions and checked whether they could be originated from the common decay vertex downstream of the main vertex. All $\Lambda$-candidates are combined with negatively charged mesons to search for decay vertices of multi-strange hyperons. The resulting invariant mass distributions for $\Xi^{-}$hyperons are presented in Fig. 5 (left). The identification of decay products of hypernuclei can be achieved by ionization energy loss (with the TPC detector) and time-of-flight (with the TOF detector) measurements. The reconstruction of secondary vertices displaced from the main interaction vertex can be achieved by using the TPC information alone. The results of simulation of $H_{\Lambda}^{3}$ production and decay, and consecutive reconstruction of invariant mass of helium-3 nuclei and charged hadrons are shown in Fig. 5 (right). 


\section{References}

[1] B. Friman, W. Nörenberg, and V.D. Toneev, Eur. Phys. J. A 3 (1998)

[2] C. Blume, J. Phys. G 31, S57 (2005)

[3] A. Andronic et al., Phys. Lett. B 695, 203 (2011)

[4] J. Steinheimer et al., Progress in Particle and Nuclear Physics 62, 313317 (2009)

[5] J. Steinheimer, K. Gudima, A. Botvina, I. Mishustin, M. Bleicher, H. Stocker, Phys. Lett. B 714, 85 (2012)

[6] NICA White Paper. http://theoro.jinr.ru/twiki-cgi/view/NICA/NICAWhitePaper

[7] BM@N Conceptual Design Report: http://nica.jinr.ru/files/BM@N/BMN_CDR.pdf

[8] D. Suvarieva, M. Ilieva, M. Kapishin, V. Kolesnikov, V. Vasendina and A. Zinchenko, J.Phys.Conf.Ser. 668 (2016) no.1, 012121

[9] V. Friese, Strangeness Prospects with the CBM Experiment, talk at the SQM-2015 Conference, Dubna, Russia, July 6-11, 2015.

[10] J. Heuser et al., Technical Design Report for the CBM Silicon Tracking System, CBM Collaboration, GSI Report 2013-4, http://repository.gsi.de/record/54798

[11] D.Baranov et al., JINST 12 (2017) no.06, C06041

[12] M.Kapishin (for the BM@N Collaboration), Eur.Phys.J. A52 (2016) no.8, 213

[13] M.Kapishin et al, PoS EPS-HEP2017 (2017) 657

[14] M.Kapishin (for the BM@N Collaboration), Phys.Atom.Nucl. 80 (2017) no.10, 1613-1619, Yad.Fiz. 7 (2016) no.6, 543-550

[15] V. Akishina and I. Kisel, J. Phys.: Conf. Ser. 599, 012024 (2015), I. Kisel, Nucl. Instrum. Meth. A 566, 85 (2006)

[16] D.Baranov et al., Phys.Part.Nucl.Lett. 15 (2018) no.2, 148-156

[17] SRC project at BM@N:

http://bmnshift.jinr.ru/wiki/lib/exe/fetch.php?media=proposal_bmn_dubna_final.pdf

[18] V. Kekelidze, A. Kovalenko, R. Lednicky, V. Matveev, I.Meshkov, A. Sorin, G. Trubnikov, 37th International Conference on High Energy Physics, ICHEP 2014, Valencia, Spain, 2 Jul 2014 - 9 Jul 2014 Nuclear physics B Proceedings supplements (2014).

[19] V. Kekelidze, A. Kovalenko, R. Lednicky, V. Matveev, I. Meshkov, A. Sorin, G. Trubnikov; Nucl.Phys. A956 (2016) 846-849.

[20] Kh.U. Abraamyan et al.. Nucl.Instrum.Meth. A628 (2011) 99-102.

[21] V. Golovatyuk, V. Kekelidze, V. Kolesnikov, O. Rogachevsky, A. Sorin; Eur.Phys.J. A52 (2016) no.8, 212.

[22] Topical Issue on Exploring Strongly Interacting Matter at High Densities - NICA White Paper, EPJ 52 (2016) (edited by D. Blaschke et al.).

[23] J. Cleymans, Phys. Part. Nucl. Lett., Vol. 8, No. 8, 797 (2011).

[24] O. Rogachevsky, A. Sorin, O. Teryaev, Phys. Rev. C 82, 054910, 2010.

[25] M. Baznat, K. Gudima A. Sorin, O. Teryaev, Phys. Rev. C97, 041902, 2018. 